\title{
Image Cognizance through Fuzion - Blending
}

\author{
Disha Suru \\ Student M.E.(EXTC) \\ D. J. Sanghvi College of \\ Engineering, Mumbai
}

\author{
Sunil Karamchandani \\ Assistant Professor \\ D. J. Sanghvi College of \\ Engineering, Mumbai
}

\begin{abstract}
High dynamic range (HDR) imaging from a set of sequential exposures is not an easy way to capture high quality images of static scenes due either observational constraints or design constraints. It also suffers from artifacts for scenes with significant motion. In this paper, we proposed a new approach to HDR reconstruction that not only draws information but also it is more robust to camera scene motion than previous techniques because it simply utilize single image. As proposed method based on single image fusion, alignment process is completely neglected. This allows us to produce and HDR result which is more accurately represent the range of intensity levels found in real scenes, ranging from direct sunlight to faint starlight. The main aim of this is to reproduce what the human eye can see in terms of exposure and that offer a heightened view of reality with grater saturation and tonal range.
\end{abstract}

\section{Keywords}

Blending, destination image, High Dynamic Range (HDR), source image

\section{INTRODUCTION}

In image processing and computer graphics, high dynamic range images enable one to record a greater range of tonal detail than a camera could capture in a single image. However, the human eye can see a lot broader dynamic range (bright and darks) at the same time than a camera can. This is because the human eye can vary its exposure sensitivity locally in just one area rather than only across the whole image. This allows us to see the inside of a darkened room as well as the brightly lit world out the window at the same time. Digital cameras have a limited dynamic range, which is lower than one encounter in the real world. We have a number of situations in medical stream where we need a broader dynamic range than the equipment allows. High-dynamicrange images are generally achieved by capturing multiple standard images, often using exposure bracketing, and then merging them into an HDR image[1]-[3]. Upon display, the intensities need to be remapped to match the typically low dynamic range of the display device, through a process of tone mapping. The effect is amazing if done with proper efforts. The proposed method is a simpler way to create the effect of an HDR image, using only one image resulting into a high-quality, low dynamic range image, ready for display. This creates effects similar to HDR image.

\section{MOTIVATION}

The instruments (camera etc) are not capable of providing such information either by design or because of observational constraints. Observational constraints and design constraints should be relative motion to each other. It is easy to capture high-quality image of static scenes but it suffers from artifacts for scenes with significant motion. It is incapability of instruments, which unable to capture brighter and darker area of scene simultaneously. Either the scene is over exposure or under exposure. Under exposure area results in darker view in the scene and on the other hand over exposure area results in brighter patch in the image, actual condition of scene is not capture. This is main drawback for medical stream where actual condition of image really matters [4],[5]. As per latest research done by Microsoft at Harvard University, the major challenge in image fusion is combining large images set from photos to produce as result that uses the best parts of the images to create more informative image [6]. Image Registration is a pre processing stage of all image fusion techniques. It is the major source of error in image fusion [7]. As per above discussion capturing more than one picture at a time is difficult and combing all together is major challenge. To remedy it we are using single image, split it into two images and further they are fused by image blender so resultant image is well exposure. As whole process is done on single image, image alignment process can simply be avoided, error is reduced. This will be very helpful in medical stream.

\section{PROPOSED METHOD}

\subsection{Algorithm Description}

1. Create Image Blender: Convert RGB image into HLS (Hue, Luminosity and saturation) color space and then recombined and converted back into RGB space using luminosity and saturation of destination pixel and hue of source pixel.

2. Load original image

3. Display the original

4. Create a copy of image

5. Convert it to grayscale -Desaturate the image

By desaturation, One can convert all the colors of the image to corresponding shades of gray. This differ from converting the image to grayscale because the RGB values with three components are still presvered. Desaturation or Luminosity is estimated by NTSC defined color weight [8].

The gray levels will be calculated as by using (1),

Luminosity $=0.21 \times R+0.72 \times G+0.07 \times B$

\section{Invert the image}

Here, we are multiplying color matrix with inverted matrix to get maximum tonal information [9].

ColorMatrix $=$ new ColorMatrix $($ new float [][] new float $\left[\begin{array}{lllll}-1.0, & 0.0, & 0.0, & 0.0, & 0.0\end{array}\right\}$, new float $\left[\begin{array}{lllll}-1.0 .0,-1.0, & 0.0, & 0.0, & 0.0\end{array}\right\}$, new float $]\left\{\begin{array}{llll}0.0, & 0.0,-1.0, & 0.0, & 0.0\end{array}\right\}$, new float $[$ ] $0.0, \quad 0.0,0.0, \quad 1.0,0.0\}$, new float $\left[\begin{array}{llll}\{1.0,1.0,1.0, & 0.0,1.0\end{array}\right\}$, \});

7. Display intermediate step in picture two.

8. Blend images using SoftLight function. 
In this step blending of two images is done with the help of various blending modes [10]. The bitmap has information in form of pixels and each pixel has three or sometimes four color channel i.e. (Red, Green, Blue and Alpha). Alpha channel also known as opacity channel which describe intensity of bitmap. So we have defined two basic functions. First function works on per-channel of the pixels. It takes every channel of the source image and destination image and applies per-channel blending function on it. Second function works on per-pixel of bitmap. It takes pixel value of source image and destination image then blend this two values by using per-pixel blending function.

\subsubsection{Per-channel Function}

\subsubsection{Blend Darken}

It creates a pixel that retains the smallest components of the source and destination pixels. If the source pixel has the components $\mathrm{r} 1, \mathrm{~g} 1$, and $\mathrm{b} 1$, and the destination has $\mathrm{r} 2, \mathrm{~g} 2, \mathrm{~b} 2$, the resultant pixel is $[\min (\mathrm{r} 1, \mathrm{r} 2), \min (\mathrm{g} 1, \mathrm{~g} 2), \min (\mathrm{b} 1, \mathrm{~b} 2)]$

\subsubsection{Blend_Multiply}

It multiplies information in each channel of source image with destination image.

\subsubsection{Blend_ColorBurn}

In this mode, destination bitmap inverted, divided by source bitmap and the quotient is then inverted.

\subsubsection{Blend_Lighten}

It has the opposite action of Darken mode. It selects the maximum of each component from the source pixel and destination pixels. The mathematical expression for lighten mode is $[\max (\mathrm{r} 1, \mathrm{r} 2), \max (\mathrm{g} 1, \mathrm{~g} 2), \max (\mathrm{b} 1, \mathrm{~b} 2)]$

\subsubsection{Blend_Screen}

This mode can be used for making blacks disappear while keeping the whites, and for making glow effects. In this mode source and destination bitmap is inverted, and the product is inverted again.

\subsubsection{Blend_ColorDodge}

In this destination bitmap is divided by inverted source bitmap.

\subsubsection{Blend_Overlay}

Overlay combines Multiply and Screen blend modes. The parts of the source where destination is light become lighter, the parts where the source is dark become darker. Function can be written by using (2),

$$
\begin{aligned}
f(a, b)= & \{2 a b \text { if } a<0.5 \\
& \{1-2(1-a)(1-b), \text { otheriwse }(2)
\end{aligned}
$$

Where $a$ is the source value and $b$ is the destination value. Depending on the value of the source, one gets a linear interpolation between black $(a=0)$, the source $(a=0.5)$, and white $(a=1)$.

\subsubsection{Blend_SoftLight}

This is a softer version of hard Light.

\subsubsection{Blend_HardLight}

It combines Multiply and Screen blend modes. Equivalent to Overlay, but with the destination and source images swapped.

\subsubsection{Blend_PinLight}

It replaces the colors, depending on destination color. If destination ( Light source) is lighter than $50 \%$ gray, pixels darker than the destination color are replaced, and pixels lighter than the destination color do not change.

\subsubsection{Blend_Difference}

Looks at the color information in each channel and subtracts either the destination color from the source color or the source color from the destination color, depending on which has the greater brightness value. Blending with white inverts the source color values; blending with black produces no change.

\subsubsection{Blend_Exclusion}

Creates an effect similar to but lower in contrast than the Difference mode.Blending with white inverts the source color values. Blending with black produces no change. One of main utilities for this is when it can be used to verify alignment of pictures with similar content.

\subsubsection{Per-Pixel Function}

\subsubsection{Blend_Hue}

The Hue blend mode preserves the luminosity and saturation of the destination, while adopting the hue of the source image.

\subsubsection{Blend_Saturation}

The Saturation blend mode preserves the Luminosity and Hue of the destination, while adopting the Saturation of the source image.

\subsubsection{Blend_Color}

The Color blend mode preserves the Luminosity of the destination, while adopting the Hue and Saturation of the source image.

\subsubsection{Blend_Luminosity}

The Luminosity blend mode preserves the Hue and Saturation of the destination, while adopting the luminosity of the source image.

9. Add some brightness to the image

$$
\begin{aligned}
& \text { ColorMatrix }=\text { new ColorMatrix (new float }[][]\{ \\
& \text { new float [ ] }\left\{\begin{array}{lllll}
1.0 & 0.0, & 0.0, & 0.0, & 0.0
\end{array}\right\} \text {, }
\end{aligned}
$$

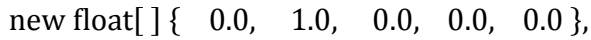

$$
\begin{aligned}
& \text { new float [ ] }\left\{\begin{array}{lllll}
0.0 & 0.0, & 1.0, & 0.0 & 0.0
\end{array}\right\} \text {, } \\
& \text { new float [ ] }\left\{\begin{array}{llll}
0.0 & 0.0,0.0, & 1.0,0.0
\end{array}\right\} \text {, }
\end{aligned}
$$

new float $[$ \{ AdjValueR, AdjValueG, AdjValueB, 0.0, 1.0$\}$, ) );

Here, simply brightness is adjusted. Brightness matrix translates colors in each channel by specified values. -1.0 reperesent complete darkness(Black), 1.0 repesents pure white color [11].

10. Display the result 


\subsection{Concept of Blending}

Blending is very simple concept. One take a pixel from source image and a pixel from destination image and combine them together using some set of rules or a formula [12]. There are basically only two types of situations possible. First is when the same formula applies to every channel of the image (red, green and blue). In this case, the function takes source byte and destination byte and returns the resulting byte. Second type of function is one that takes all the RGB data for both source and destination pixels and calculates the resulting $R, G$ and $B$ values. Resulting values are returned in destination $R$, $\mathrm{G}$ and $\mathrm{B}$ parameters.

\subsection{Application of blending functions}

In order to apply any of the functions described above to images, we have defined two separate processing functions, one each type of blending function. First one applies any specified per-channel processing function to blend each channel of the destination and source images. In this specified areas of the source and destination images are locked with LockBits methods and then processes byte by byte, so basically part of source and destination are first locked on which blending is applied. Different per-channel function is passed as the last parameter and is applied to each channel of each pixel of the source and destination images. After processing lock bits are released and finally replace the bits in the bitmap with the modified data. Second function does almost the same thing, but it takes second type of blending functions as a parameter and it process data on pixel at the time.

\section{RESULT AND DISCUSSION}

All results were constructed from JPEG- encoded images. Proposed method works on each and every size of image because it is real time based application. Fig. 1 is the source image. Fig. 2 - Fig. 6 represents five important blending modes i.e. Multiply mode, colorDodge mode, overlay mode, Difference mode and luminosity mode respectively. Result may vary as per source image. As per the quantitative analysis described in Table no.1, all blending modes results into more informative image. Multiply mode and luminosity mode provide better result while the result under difference mode is less accurate. Which blending mode is applicable on particular image is completely depend on application.

\section{CONCLUSION AND FUTURE SCOPE}

Proposed method is simple as it eliminates need of image registration. It is always not possible to capture more than one image at a time where as proposed method work on single image, this can be treated as major advantage in the medical stream. Depending upon type of the application and the requirement of user that one might have desire of the visually beautiful image at the same time someone else may require the more detailed as well as accurate results about the image, selection of blending mode as per desire application is in the hand of the user. Development and improvement are the future aspect of image fusion which can be achieved by estimating and evaluating the quality of a fused image. It is possible in future that one can try to create its own Raster operation functions instead of defined blending modes.

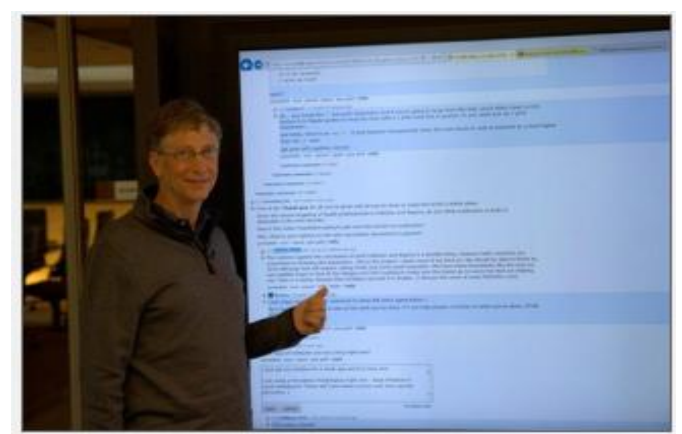

Fig. 1 Source Image

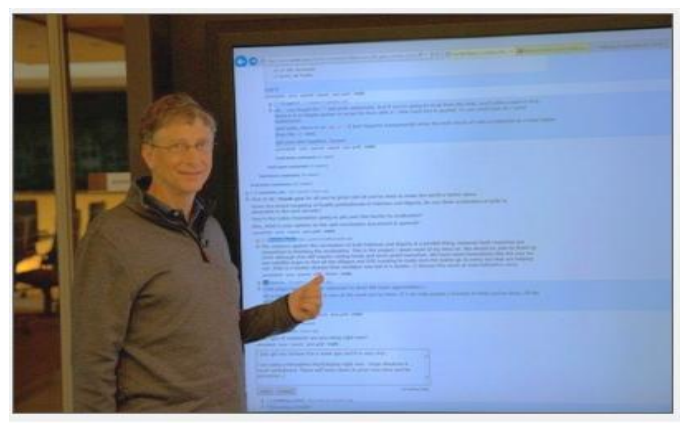

Fig. 2 Multiply Mode

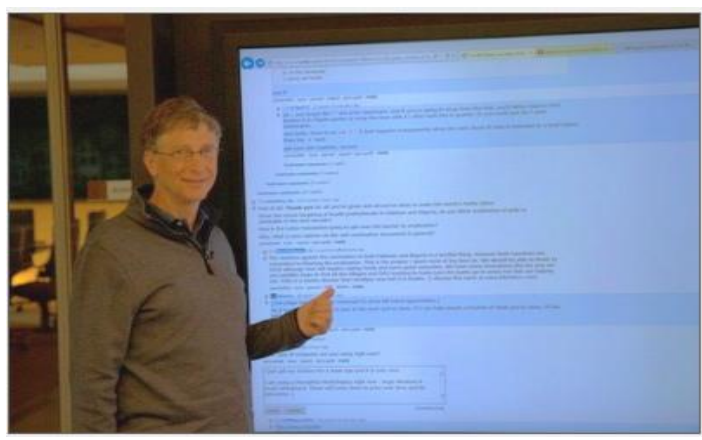

Fig. 3 ColorDodge Mode

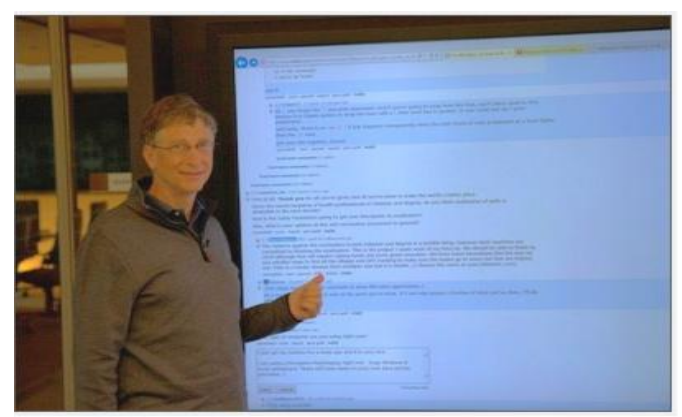

Fig. 4 Overlay Mode 


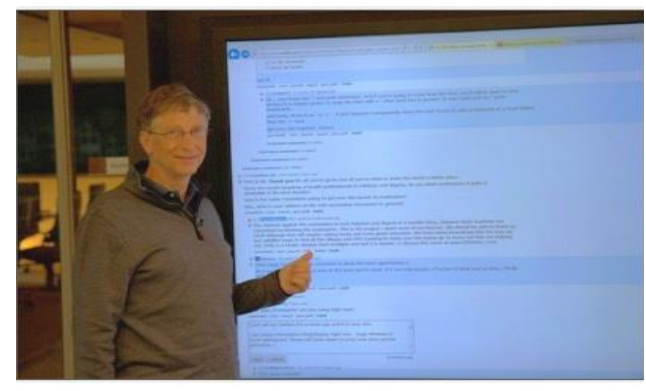

Fig. 5 Difference Mode

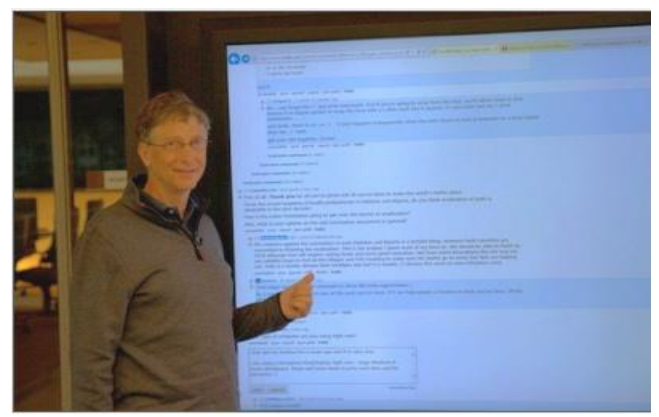

Fig. 6 Luminosity Mode

Table 1: Quantitative analysis of source and fused image results.

\begin{tabular}{|c|c|}
\hline Blending Modes & Entropy \\
\hline Source Image & 1.4530 \\
\hline Multiply mode & 2.0976 \\
\hline Color Dodge mode & 2.0594 \\
\hline Overlay mode & 2.0700 \\
\hline Difference mode & 2.0395 \\
\hline Luminosity mode & 2.0727 \\
\hline
\end{tabular}

\section{REFERENCES}

[1] P. E. Debevec and J. Malik. Recovering high dynamic range Radiance maps from photographs.In SIGGRAPH '97:Proceeding of the $24^{\text {th }}$ annual conference on Computer Graphics and interactive techniques, pages 369-378, New York,NY, USA, 1997. ACM Press/Addison- Wesley Publishing Co.

[2] S. Mann and R. Picard. Being 'undigital' with digitalCameras: Extending dynamic range by combining differently exposed pictures. In Proceedings of IS \&T 46th annual conference. pages 422-428, May 1995.

[3] E. Reinhard, G. Ward, S. Pattanaik, and P. Debevec. High Dynamic Range Imaging: Acquisition, Display and Image-Based Lighting. Morgan Kaufmann Publishers, Dec. 2005.

[4] Vavilin, K. Jo, "Fast HDR image generation frommulti-exposed multiple-view LDR images", IEEE Trans., vol. 4 pp. 105-110, July 2011.

[5] Y. Bandoh, Qui Guoping, M. Okuda, S. Dal, "Recent Advances in High Dynamic Range imaging technology," IEEE Trans.Image processing, vol.11, pp 3125-3128. , Sept 2010 .

[6] Jung Gap Kuk, Nam Ik Cho, Sang Uk Lee, "High Dynamic Range imaging by Gradient domain fusion," IEEE Trans. Image Process., vol. 10, no. 5, pp. 14611464, May 2011.

[7] J. DiCarlo and B. Wandell. Rendering high dynamic range images,. In Proceedings of SPIE, volume 3965 , Jan.2000.

[8] Shinnichi Kimura, Masato Terakura, Hirotaka Sawada, HighPerformance Visual Monitoring System For IKAROS,Advances in Microelectronic Engineering (AIME) Volume Issue 3, July 2013

[9] Yan Wang, "Fast clear single underwater image", IEEE Trans. Computational Intelligence and software engineering, pp. 1-4, Dec 2010.

[10] Yunpeng Ma, " The mathematic magic of blend modes For image processing", IEEE Trans .on multimedia Technology, pp. 5159-5161, July 2011.

[11] H. Ibrahim, N.S.P. Kang,“ Brightness preserving dynamic histogram equalization for image contrast enhancement”,IEEE Trans. on vol. 53, pp. 17521758, Nov 2007.

[12] R.T. Whitaker, " A level-set approach to image blending”,IEEE Trans. on vol. 9, pp. 1849-1861, Nov 2000. 